\title{
On the Circular Chromatic Number of Circular Partitionable Graphs
}

\author{
Arnaud Pêcher \\ Laboratoire Bordelais de Recherche Informatique (LaBRI) \\ 351 cours de la Libération, 33405 Talence, France \\ pecher@labri.fr \\ Xuding Zhu \\ Department of Applied Mathematics \\ National Sun Yat-sen University \\ Kaohsiung, Taiwan \\ zhu@math.nsysu.edu.tw
}

\begin{abstract}
This paper studies the circular chromatic number of a class of circular partitionable graphs. We prove that an infinite family of circular partitionable graphs $G$ have $\chi_{c}(G)=\chi(G)$. A consequence of this result is that we obtain an infinite family of graphs $G$ with the rare property that the deletion of each vertex decreases its circular chromatic number by exactly 1 .
\end{abstract}

Key words: circular coloring, chromatic number, partitionable graph

\section{Introduction}

Suppose $G$ is a graph and $k \geq 2 d$ are positive integers. A $(k, d)$-coloring of $G$ is a mapping $f: V(G) \rightarrow \mathbb{Z}_{k}$ such that for each edge $x y$ of $G, d \leq|f(x)-f(y)| \leq$ $k-d$. The circular chromatic number of $G$ is defined as

$$
\chi_{c}(G)=\inf \{k / d: G \text { has a }(k, d) \text {-coloring }\} .
$$

1 Supported in part by the National Science Council of ROC under grant NSC91-2115-M110-004 
Note that a $(k, 1)$-coloring of $G$ is equivalent to a $k$-coloring of $G$. Recall that the chromatic number $\chi(G)$ of $G$ is the minimum $k$ for which $G$ has a $k$-coloring. Therefore $\chi_{c}(G) \leq \chi(G)$. On the other hand, it is known [11,13] that $\chi_{c}(G)>$ $\chi(G)-1$. Therefore $\chi(G)=\left\lceil\chi_{c}(G)\right\rceil$. So the parameter $\chi_{c}(G)$ is a refinement of $\chi(G)$, and $\chi(G)$ is an approximation of $\chi_{c}(G)$.

This paper studies the circular chromatic number of partitionable graphs. There are two motivations for the study of this problem. One concerns the circular chromatic number of induced graphs. Suppose $G$ is a graph with $\chi(G)=n$. Then it is obvious that for any integer $k, G$ has an induced subgraph $H$ with $\chi(H)=k$ if and only if $k \leq n$. Given a graph $G$ with $\chi_{c}(G)=p / q$. For what numbers $r, G$ has a subgraph $H$ with $\chi_{c}(H)=r$ ? This naive question turns out to be very difficult. Instead of finding a complete list of all such numbers $r$, one may ask if some particular numbers $r$ are the circular chromatic numbers of induced subgraphs of $G$. One question, asked in [12] is that whether every graph $G$ has a vertex $v$ such that $\chi_{c}(G)-\chi_{c}(G-v) \leq 1$. Recently the question has been answered in [15] in the negative, where an infinite family of graphs $G$ was found to have $\chi_{c}(G)=4$ and $\chi_{c}(G-v)=8 / 3$ for each vertex $v$ of $G$. Another particular question, asked in [13], is that which graphs $G$ have the property that the deletion of any vertex decreases its circular chromatic number by exactly 1 ? Complete graphs, graphs which are the direct sum of two $\chi$-critical graphs, and some other isolated example graphs are known to have this property. In general, this seems to be a rare property. To search for graphs with this property, partitionable graphs are natural candidates. We shall prove that for an infinite family of partitionable graphs $G$ we have $\chi_{c}(G)=\chi(G)$. Because $G$ is a partitionable graph, for any vertex $v$, $\chi(G-v)=\omega(G-v)=\chi(G)-1$, which implies that $\chi_{c}(G-v)=\chi(G)-1$. Therefore for these partitionable graphs, the deletion of any vertex decreases its circular chromatic number by exactly 1 .

Another motivation concerns the study of circular perfect graphs. Given positive integers $k \geq 2 d$, the circular complete graph $K_{k / d}$ has vertex set $\mathbb{Z}_{k}$ in which $i j$ is an edge if $d \leq|i-j| \leq k-d$. A homomorphism from a graph $G$ to a graph $H$ is a mapping $f: V(G) \rightarrow V(H)$ such that $f(x) f(y)$ is an edge of $H$ whenever $x y$ is an edge of $G$. Then a $k$-coloring of a graph $G$ is equivalent to a homomorphism from $G$ to $K_{k}$ and a $(k, d)$-coloring of $G$ is equivalent to a homomorphism from $G$ to $K_{k / d}$. So in the study of circular chromatic number of graphs, the circular complete graphs $K_{k / d}$ play the role of complete graphs as in the study of chromatic number of graphs. The circular clique number of a graph $G$ is defined as

$$
\omega_{c}(G)=\sup \left\{k / d: K_{k / d} \text { admits a homomorphism to } G\right\} \text {. }
$$

It was shown in [14] that $\omega_{c}(G)$ is equal to the maximum of those $k / d$ for which $K_{k / d}$ is an induced subgraph of $G$.

A graph $G$ is called circular perfect [14] if for every induced subgraph $H$ of $G$, $\chi_{c}(H)=\omega_{c}(H)$. Since $\omega(H) \leq \omega_{c}(H) \leq \chi_{c}(H) \leq \chi(H)$, every perfect graph 
is circular perfect. On the other hand, there are circular perfect graphs which are not perfect. In particular, odd cycles and the complement of odd cycles are circular perfect graphs. So the class of circular perfect graphs is strictly larger than the class of perfect graphs, but it is still a very restrictive class. A sufficient condition for a graph to be circular perfect is given in [14], and this sufficient condition is used to prove an analogue of Hajos Theorem for circular chromatic number. We believe that the class of circular perfect graphs is an interesting class of graphs, although the concept is quite new, and not much is known about it. We call a graph $G$ minimal circular imperfect, if $G$ is not circular perfect but every proper induced subgraph of $G$ is circular perfect. As an analogue to the study of perfect graphs, it would be nice to have an appealing conjecture on the structure of minimal circular imperfect graphs.

As all the minimal imperfect graphs are circular perfect, we need to search a larger area for minimal circular imperfect graphs. The class of partitionable graphs is again a natural candidate. This paper proves that none of a subclass of partitionable graphs is a minimal circular imperfect graph.

\section{Circular partitionable graphs and the main result}

Suppose $p, q \geq 2$ are integers. A graph $G$ is a $(p, q)$-partitionable graph if $|V(G)|=$ $p q+1$, and for each vertex $v$ of $G, G \backslash\{v\}$ admits a partition into $p$ cliques of cardinality $q$ as well as a partition into $q$ stable sets of cardinality $p$. A graph is partitionable if it is a $(p, q)$-partitionable graph for some $p, q \geq 2$. Partitionable graphs were introduced by Lovász [9] and Padberg [10] as a tool in the study of perfect graphs. A graph $G$ is perfect if for every induced subgraph $H$ of $G$, we have $\chi(H)=\omega(H)$. Here $\omega(H)$ is the clique number of $G$, which is the cardinality of a maximum clique of $G$. A graph $G$ is minimal imperfect if $G$ is not perfect, but every induced subgraph of $G$ is perfect. The Strong Perfect Graph Theorem, which was conjectured by Berge [2] in 1961, proved by Chudnovsky, Robertson, Seymour and Thomas [5] in 2002, says that odd cycles of length at least 5 and their complements are the only minimal imperfect graphs. Before the proof of Berge's conjecture, it was shown by Lovász [9] and Padberg [10] that every minimal imperfect graph is a partitionable graph. Thus to prove Berge's conjecture, it suffices to show that none of the partitionable graph is a counterexample. Although the final proof of Berge's conjecture given by Chudnovsky, Robertson, Seymour and Thomas [5] takes a different route, the class of partitionable graphs has been studied thoroughly in the literature, and this turns out to be an interesting class of graphs. The understanding of the structure of this class of graphs may be helpful in the study of other graph theory problems. It is known (cf. [3]) that a $(p, q)$-partitionable graph has the following properties:

(1) $p$ is the maximum cardinality of a stable set of $G$, and $q$ is the maximum car- 
dinality of a clique of $G$;

(2) $G$ has exactly $n$ stable sets of cardinality $p$ and exactly $n$ cliques of cardinality $q$, where $n$ is the number of vertices of $G$;

(3) For each maximum clique $C$ of $G$, there is a unique maximum stable set $S$ such that $C \cap S=\emptyset$; and similarly, for each maximum stable set $S$ there is a unique maximum clique $C$ such that $C \cap S=\emptyset$;

(4) Each vertex belongs to exactly $q$ maximum cliques, and belongs to $p$ maximum stable sets.

In the study of partitionable graphs, some recursive constructions of sub-families of partitionable graphs are discussed in the literature $[6,4]$. The class of circular partitionable graphs was introduced by Chvátal, Graham, Perold and Whitesides [6].

For two sets of integers $X, Y$, let $X+Y$ denote the set $\{x+y: x \in X, y \in Y\}$. If $X=\{x\}$ is a singleton, we write $x+Y$ instead of $\{x\}+Y$.

Suppose $m_{i} \geq 2(i=1,2, \cdots, 2 r)$ are integers. Define integers $\mu_{i}$ (for $i=$ $0,1, \cdots, 2 r$ ), sets $M_{i}$ (for $i=1,2, \cdots, 2 r$ ), and sets $C, S$ as follows:

$$
\begin{aligned}
\mu_{i} & =m_{1} m_{2} \cdots m_{i}\left(\mu_{0}=1\right) \\
M_{i} & =\left\{0, \mu_{i-1}, 2 \mu_{i-1}, \cdots,\left(m_{i}-1\right) \mu_{i-1}\right\}, \\
C & =M_{1}+M_{3}+\cdots+M_{2 r-1} \\
S & =M_{2}+M_{4}+\cdots+M_{2 r}
\end{aligned}
$$

Let $n=m_{1} m_{2} \cdots m_{2 r}+1$. We denote by $C\left[m_{1}, m_{2}, \cdots, m_{2 r}\right]$ the circulant graph with vertex set $\mathbb{Z}_{n}=\{0,1, \cdots, n-1\}$, where $x y$ is an edge if and only if $x \neq y$ and $x-y$ modulo $n$ is equal to the difference of two elements of $C$.

Note that $\mu_{i}>\max \sum_{j=1}^{i-1} M_{j}$. This implies that $|C|=m_{1} m_{3} \cdots m_{2 r-1}$ and $|S|=$ $m_{2} m_{4} \cdots m_{2 r}$. Let $\omega=|C|$ and $\alpha=|S|$. Then $n=\omega \alpha+1$. Suppose $X$ is a subset of $\mathbb{Z}_{n}$. A circular shift of $X$ is a set of the form $i+X=\{i+x(\bmod n): x \in X\}$.

Theorem 1 [6] Suppose $m_{i} \geq 2$ are integers for $i=1,2, \cdots, 2 r$. Then $G=$ $C\left[m_{1}, m_{2}, \cdots, m_{2 r}\right]$ is an $(\alpha, \omega)$-partitionable graph. Moreover, the $n$ maximum cliques of $G$ are the $n$ circular shifts of $C$, and the $n$ maximum stable sets of $G$ are the $n$ circular shifts of $S$.

As an example, we consider the graph $C[2,2,2,2]$. Then 


$$
\begin{aligned}
\mu_{i} & =2^{i}, i=0,1,2,3,4 ; \\
M_{1} & =\{0,1\}, M_{2}=\{0,2\}, M_{3}=\{0,4\}, M_{4}=\{0,8\} \\
C & =\{0,1,4,5\} \\
S & =\{0,2,8,10\} .
\end{aligned}
$$

The vertex set of $C[2,2,2,2]$ is $\mathbb{Z}_{17}$, and $i j$ is an edge if $|i-j| \in\{1,3,4,5\}$. The graph is depicted in Figure 2.

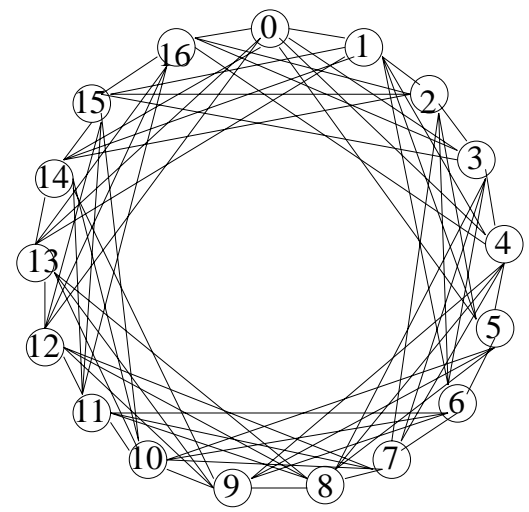

Fig. 1. The circular partitionable graph $C[2,2,2,2]$

The following is the main result of this paper.

Theorem 2 Let $x_{1}, \ldots, x_{p}(p \geq 2)$ be integers such that $x_{i} \geq 2$ for every $1 \leq i \leq$ $p$. Let $\delta=\max x_{i}$ and let $G=C\left[x_{1}, 2, \ldots, x_{p}, 2\right]$. If $p=2$ or $x_{1} x_{2} \ldots x_{p} \geq 2^{p+1} \delta$ then $\chi_{c}(G)=\chi(G)$.

The proof of Theorem 2 is left to Section 4.

Observe that for $G=C\left[x_{1}, 2, \ldots, x_{p}, 2\right], \alpha=2^{p}, \omega=x_{1} x_{2} \cdots x_{p}$ and $|V(G)|=$ $n=2^{p} x_{1} x_{2} \ldots x_{p}+1$. Therefore

$$
\chi_{f}(G)=\frac{n}{\alpha}=\omega+\frac{1}{2^{p}}<\chi_{c}(G)=\chi(G)=\omega+1 .
$$

There are a few papers devoted to the study of the circular chromatic number of circulant graphs [7,8]. It is known [13] that for any graph $G, \chi_{f}(G) \leq \chi_{c}(G) \leq$ $\chi(G)$. A graph $G$ is called star extremal if $\chi_{f}(G)=\chi_{c}(G)$. In [7,8], classes of star extremal circulant graphs are investigated. Theorem 2 provides a class of circulant graphs of another kind of extremality, i.e., circulant graphs with $\chi_{c}(G)=\chi(G)$.

Corollary 3 Suppose $G=C\left[x_{1}, 2, \ldots, x_{p}, 2\right]$ satisfies the condition of Theorem 2. Then $G$ is circular imperfect.

PROOF. It is known [14] that $\omega(G) \leq \omega_{c}(G)<\omega(G)+1$. As $\chi_{c}(G)=\chi(G)=$ $\omega(G)+1$, it follows that $\chi_{c}(G)>\omega_{c}(G)$. 
It is unknown if any of the graphs $G=C\left[x_{1}, 2, \ldots, x_{p}, 2\right]$ are minimal circular imperfect. A computer search shows that $C[2,2,2,2]$ is not minimal circular imperfect. The subgraph of $C[2,2,2,2]$ induced by the set $X=\{2,3,4,5,6,9,11$, $12,13,14,16\}$ has circular chromatic number 4 and circular clique number 3 , and hence is circular imperfect.

Since the graphs $G=C\left[x_{1}, 2, x_{2}, 2, \cdots, x_{p}, 2\right]$ are partitionable, $\chi(G-x)=$ $\omega(G-x)=\chi_{c}(G-x)=\chi(G)-1$ for each vertex $x$. Therefore we have the following corollary.

Corollary 4 Suppose $G=C\left[x_{1}, 2, \ldots, x_{p}, 2\right]$ satisfies the condition of Theorem 2. Then $\chi_{c}(G-x)=\chi_{c}(G)-1$ for each vertex $x$ of $G$.

In other words, the circulant graphs satisfying the condition of Theorem 2 have the property that the deletion of each vertex decreases its circular chromatic number by exactly 1 . Not many such graphs were known before, and the problem of characterizing and constructing such graphs was raised in [13].

\section{Structural properties of $G$}

In the remainder of this paper, $G=C\left[x_{1}, 2, x_{2}, \ldots, x_{p}, 2\right]$. Let $\omega=x_{1} \ldots x_{p}$, $\alpha=2^{p}, n=\alpha \omega+1$.

In this section, we shall be interested in elements of $\mathbb{Z}_{n}$ only. If $a \equiv b(\bmod n)$, then $a, b$ are treated as the same. However, by an abuse of notation, we also use the natural order of integers in the following sense: If $a<b$, we denote $[a, b]$ the set of integers $a \leq x \leq b$. Note that it is possible that $a, b \notin\{0,1, \cdots, n-1\}$. However, $[a, b]$ always denote a subset of $\{0,1, \cdots, n-1\}$, by means of taking modulo $n$. For example, $[-1,1]=\{-1,0,1\}=\{n-1,0,1\}$. For a set $A$ of integers, let $a A=\{a x: x \in A\}, a+A=\{a+x: x \in A\}$. Again the multiplications and additions are modulo $n$.

Let $c_{0}=1$, and for $i=1, \ldots, p$, let

$$
c_{i}=2^{i-1} x_{1} x_{2} \ldots x_{i}, \text { and } d_{i}=\sum_{j=1}^{i} c_{j} .
$$

First we derive an explicit expression of the maximum cliques and stable sets of $G$. It follows from the definition that $\mu_{0}=1$ and for $1 \leq i \leq p$,

$$
\mu_{2 i-1}=2^{i-1} x_{1} x_{2} \ldots x_{i}=c_{i} \text {, and } \mu_{2 i}=2^{i} x_{1} x_{2} \ldots x_{i}=2 c_{i} .
$$


Therefore for $1 \leq i \leq p$,

$$
M_{2 i-1}=2 c_{i-1}\left[0, x_{i}-1\right], \text { and } M_{2 i}=\left\{0, c_{i}\right\}
$$

So

$$
\begin{aligned}
C & =\sum_{j=1}^{p} M_{2 j-1}=\sum_{j=1}^{p} 2 c_{j-1}\left[0, x_{j}-1\right], \\
S & =\sum_{j=1}^{p} M_{2 j}=\sum_{j=1}^{p}\left\{0, c_{j}\right\} .
\end{aligned}
$$

By Theorem 1, the maximum cliques of $G$ are the $n$ circular shifts $C+i$ of $C$ $\left(i \in \mathbb{Z}_{n}\right.$ ), and the maximum stable sets of $G$ are the $n$ circular shifts $S+i$ of $S$ $\left(i \in \mathbb{Z}_{n}\right)$.

In the following, we consider the intersections $S \cap(S+i)$ of two maximum stable sets of $G$.

Lemma 5 For $2 \leq i \leq p, c_{i} \notin\left[-2 d_{i-1}, 2 d_{i-1}\right]$.

PROOF. Since $c_{2}=2 x_{1} x_{2}>2 x_{1}=2 d_{1}$, and $n-c_{2} \geq 4 x_{1} x_{2}+1-2 x_{1} x_{2}>$ $2 x_{1} x_{2}>2 d_{1}$, we conclude that $c_{2} \notin\left[-2 d_{1}, 2 d_{1}\right]$. Assume $i \geq 3$ and $c_{i-1} \notin$ $\left[-2 d_{i-2}, 2 d_{i-2}\right]$. Since $c_{i}=2 x_{i} c_{i-1}$ and $x_{i} \geq 2$, we have

$$
c_{i} \geq 2 c_{i-1}+2 c_{i-1}>2\left(2 d_{i-2}\right)+2 c_{i-1}>2 d_{i-1} .
$$

Furthermore,

$$
n-c_{i}=2^{p} x_{1} x_{2} \cdots x_{p}+1-c_{i}>2 c_{i}-c_{i}=c_{i}>2 d_{i-1} .
$$

Therefore $c_{i} \notin\left[-2 d_{i-1}, 2 d_{i-1}\right]$.

For $i=1,2, \cdots, p$, let

$$
S_{i}=\sum_{j=1}^{i} M_{2 j}=\sum_{j=1}^{i}\left\{0, c_{j}\right\} .
$$

Lemma 6 For every $x$ in $\mathbb{Z}_{n}$, for every $2 \leq i \leq p$,

$$
\begin{gathered}
S_{i} \cap\left(S_{i}+x\right)=\left(S_{i-1} \cap\left(S_{i-1}+x\right)\right) \cup\left(\left(S_{i-1}+c_{i}\right) \cap\left(S_{i-1}+c_{i}+x\right)\right) \\
\text { or } \\
S_{i} \cap\left(S_{i}+x\right)=\left(S_{i-1} \cap\left(S_{i-1}+c_{i}+x\right)\right) \cup\left(\left(S_{i-1}+c_{i}\right) \cap\left(S_{i-1}+x\right)\right) .
\end{gathered}
$$


PROOF. By definition, $S_{i}=S_{i-1} \cup\left(S_{i-1}+c_{i}\right)$. Hence $S_{i}+x=\left(S_{i-1}+x\right) \cup$ $\left(S_{i-1}+c_{i}+x\right)$. Therefore

$$
\begin{aligned}
S_{i} \cap\left(S_{i}+x\right)= & \left(S_{i-1} \cap\left(S_{i-1}+x\right)\right) \cup\left(\left(S_{i-1}+c_{i}\right) \cap\left(S_{i-1}+c_{i}+x\right)\right) \\
& \cup\left(S_{i-1} \cap\left(S_{i-1}+c_{i}+x\right)\right) \cup\left(\left(S_{i-1}+c_{i}\right) \cap\left(S_{i-1}+x\right)\right) .
\end{aligned}
$$

If $S_{i-1} \cap\left(S_{i-1}+x\right) \neq \emptyset$, then $x \in\left[-d_{i-1}, d_{i-1}\right]$. By Lemma 5, $c_{i} \notin\left[-2 d_{i-1}, 2 d_{i-1}\right]$. Therefore $c_{i}+x, c_{i}-x \notin\left[-d_{i-1}, d_{i-1}\right]$. This implies that $S_{i-1} \cap\left(S_{i-1}+c_{i}+x\right)=\emptyset$ and $\left(S_{i-1}+c_{i}\right) \cap\left(S_{i-1}+x\right)=\emptyset$. Therefore

$$
S_{i} \cap\left(S_{i}+x\right)=\left(S_{i-1} \cap\left(S_{i-1}+x\right)\right) \cup\left(\left(S_{i-1}+c_{i}\right) \cap\left(S_{i-1}+c_{i}+x\right)\right) .
$$

If $S_{i-1} \cap\left(S_{i-1}+x\right)=\emptyset$, then $\left(S_{i-1}+c_{i}\right) \cap\left(S_{i-1}+c_{i}+x\right)=\emptyset$, and hence

$$
S_{i} \cap\left(S_{i}+x\right)=\left(S_{i-1} \cap\left(S_{i-1}+c_{i}+x\right)\right) \cup\left(\left(S_{i-1}+c_{i}\right) \cap\left(S_{i-1}+x\right)\right) .
$$

For $1 \leq j \leq i \leq p$, let

$$
B_{i, j}=\sum_{t \in\{1,2, \cdots, i\} \backslash\{j\}} M_{2 t}=\sum_{t \in\{1,2, \cdots, i\} \backslash\{j\}}\left\{0, c_{t}\right\} .
$$

Then $S_{i}=B_{i, j}+\left\{0, c_{j}\right\}=B_{i, j} \cup\left(B_{i, j}+c_{j}\right)$. For convenience, let $B_{j}=B_{p, j}$.

Lemma 7 For every $1 \leq i \leq p$, for every $x \in \mathbb{Z}_{n}, x \neq 0$, we have $\left|S_{i} \cap\left(S_{i}+x\right)\right| \leq$ $2^{i-1}$. Moreover, if $\left|S_{i} \cap\left(S_{i}+x\right)\right|=2^{i-1}$, then there is a unique index $j \leq i$ such that $x= \pm c_{j}$ and $S_{i} \cap\left(S_{i}+x\right)=B_{i, j}$ or $B_{i, j}+c_{j}$, depending on $x=-c_{j}$ or $x=c_{j}$. In particular, for any $x \in \mathbb{Z}_{n},|S \cap(S+x)| \leq 2^{p-1}$, and if equality holds then there is a unique index $j$ such that $x= \pm c_{j}$ and $S \cap(S+x)=B_{j}$ or $B_{j}+c_{j}$, depending on $x=-c_{j}$ or $x=c_{j}$.

PROOF. We prove this lemma by induction on $i$. It is obvious that for every $x \in$ $\mathbb{Z}_{n}, x \neq 0$, we have $\left|S_{1} \cap\left(S_{1}+x\right)\right| \leq 1$, and equality holds only if $x= \pm c_{1}$. Let $i \geq 2$ and the lemma is true for $i^{\prime} \leq i-1$.

By Lemma 6,

$$
\begin{aligned}
& S_{i} \cap\left(S_{i}+x\right)=\left(S_{i-1} \cap\left(S_{i-1}+x\right)\right) \cup\left(\left(S_{i-1}+c_{i}\right) \cap\left(S_{i-1}+c_{i}+x\right)\right) \\
& \text { or } \\
& S_{i} \cap\left(S_{i}+x\right)=\left(S_{i-1} \cap\left(S_{i-1}+c_{i}+x\right)\right) \cup\left(\left(S_{i-1}+c_{i}\right) \cap\left(S_{i-1}+x\right)\right)
\end{aligned}
$$


First we consider the case that

$$
S_{i} \cap\left(S_{i}+x\right)=\left(S_{i-1} \cap\left(S_{i-1}+x\right)\right) \cup\left(\left(S_{i-1}+c_{i}\right) \cap\left(S_{i-1}+c_{i}+x\right)\right) .
$$

By induction hypothesis, $\left|S_{i-1} \cap\left(S_{i-1}+x\right)\right| \leq 2^{i-1}$ and $\mid\left(\left(S_{i-1}+c_{i}\right) \cap\left(S_{i-1}+\right.\right.$ $\left.\left.c_{i}+x\right)\right) \mid \leq 2^{i-1}$. Therefore

$$
\left|S_{i} \cap\left(S_{i}+x\right)\right| \leq 2 * 2^{i-1}=2^{i}
$$

Moreover, if $\left|S_{i} \cap\left(S_{i}+x\right)\right|=2^{i}$, then $\left|S_{i-1} \cap\left(S_{i-1}+x\right)\right|=2^{i-1}$ and hence there is a unique index $j \leq i-1$ such that $x= \pm c_{j}$ and $S_{i-1} \cap\left(S_{i-1}+x\right)=B_{i-1, j}$ or $B_{i-1, j}+c_{j}$. Then $S_{i} \cap\left(S_{i}+x\right)=B_{i-1, j}+\left\{0, c_{i}\right\}=B_{i, j}$ or $S_{i} \cap\left(S_{i}+x\right)=$ $B_{i-1, j}+c_{j}+\left\{0, c_{i}\right\}=B_{i, j}+c_{j}$

Next we assume that

$$
S_{i} \cap\left(S_{i}+x\right)=\left(S_{i-1} \cap\left(S_{i-1}+c_{i}+x\right)\right) \cup\left(\left(S_{i-1}+c_{i}\right) \cap\left(S_{i-1}+x\right)\right) .
$$

If $x \neq \pm c_{i}$ then the same argument as in the previous case works. Assume $x=c_{i}$. Then

$$
\left|S_{i} \cap\left(S_{i}+x\right)\right|=\left|S_{i-1}+c_{i}\right|+\left|S_{i-1} \cap\left(S_{i-1}+2 c_{i}\right)\right| .
$$

Note that if $i \leq p-1$, then $2 c_{i} \in C$. If $i=p$, then $2 c_{p}=-1$. In any case, $2 c_{i}$ is equal to the difference of two integers of $C$. Therefore $\left\{0,2 c_{i}\right\}$ is an edge of $G$. Hence $2 c_{i} \notin S_{i-1}-S_{i-1}$ (as $S_{i-1}$ is a stable set of $G$ ). This implies that

$$
S_{i-1} \cap\left(S_{i-1}+2 c_{i}\right)=\emptyset
$$

Hence

$$
\left|S_{i} \cap\left(S_{i}+x\right)\right|=\left|S_{i-1}\right|=2^{i-1},
$$

and

$$
S_{i} \cap\left(S_{i}+x\right)=B_{i, i}+c_{i}
$$

as $B_{i, i} \cap\left(B_{i, i}+2 c_{i}\right)=\emptyset\left(B_{i, i}\right.$ is a stable set and $\left\{0,2 c_{i}\right\}$ is an edge).

If $x=-c_{i}$, then the same argument shows that

$$
S_{i} \cap\left(S_{i}+x\right)=B_{i, i}
$$

\section{Proof of Theorem 2}

Assume that $\chi_{c}(G)=k / d$, where $(k, d)=1$. Let $f$ be a $(k, d)$-coloring of $G$, which is viewed as a homomorphism from $G$ to $K_{k / d}$. For $i \in \mathbb{Z}_{k}$, let $X_{i}=f^{-1}(i)$ be the set of vertices of $G$ of color $i$. Let $Y_{i}=X_{i} \cup X_{i+1} \cup \cdots \cup X_{i+d-1}$. Then 
$Y_{i}$ is a stable set. It is known (see Lemma 1.3 of [13]) that for each $i, X_{i} \neq \emptyset$ and moreover, for each $i$, there is a vertex $x \in X_{i}$ and a vertex $y \in X_{i+d}$ such that $x y$ is an edge of $G$. We need to prove that $d=1$.

Lemma 8 If $\chi_{c}(G)=k / d$ and $(k, d)=1$, then $d \leq 2$.

PROOF. Assume to the contrary that $d \geq 3$. We consider two cases.

- Case 1: $p=2$

Note that in this case $\alpha=4$.

Assume that there is an index $i$ for which $\left|X_{i}\right| \geq 2$. Since $d \geq 3, X_{i} \cup X_{i+1} \cup$ $X_{i+2} \subseteq Y_{i}$ and $X_{i-1} \cup X_{i} \cup X_{i+1} \subseteq Y_{i-1}$. However, $\left|Y_{i-1}\right|,\left|Y_{i}\right| \leq \alpha=4$, and $\left|X_{i-1} \cup X_{i} \cup X_{i+1}\right|,\left|X_{i} \cup X_{i+1} \cup X_{i+2}\right| \geq 4$ (as $\left|X_{i}\right| \geq 2$, and each $\left|X_{j}\right| \geq 1$ ). Therefore $\left|Y_{i-1}\right|=\left|Y_{i}\right|=4$, and hence $Y_{i-1}, Y_{i}$ are maximum stable sets of $G$. However, $\left|Y_{i-1} \cap Y_{i}\right|=\left|X_{i}\right|+\left|X_{i+1}\right| \geq 3>\alpha / 2$, in contrary to Lemma 7 .

Hence, for every $i \in \mathbb{Z}_{k}$, we have $\left|X_{i}\right|=1$. In particular, $n=k=4 \omega+1$. From $\frac{k}{d} \leq \chi=\omega+1$ and $\omega>2$, we get $d \geq 4$. Since $Y_{0}$ is a stable set of size $d$, we have $d=4$. Thus $Y_{0}$ and $Y_{1}$ are two maximum stable sets of $G$ sharing $d-1$ vertices, in contrary again to Lemma 7.

This completes the proof of Case 1 .

- Case 2: $\omega \geq 2^{p+1} \delta$

If there exists $i \in \mathbb{Z}_{k}$ such that $\left|Y_{i}\right|+\left|Y_{i+1}\right|+\left|Y_{i+2}\right|=3 \alpha$ then $\left|Y_{i}\right|=\left|Y_{i+1}\right|=$ $\left|Y_{i+2}\right|=\alpha$. By Claim 7, we have $\left|Y_{i} \cap Y_{i+1}\right| \leq \frac{\alpha}{2}$. Hence $\left|Y_{i+1} \backslash Y_{i}\right| \geq \frac{\alpha}{2}$. As $Y_{i+1} \backslash Y_{i}=X_{i+d}$, we get $\left|X_{i+d}\right| \geq \frac{\alpha}{2}$. As $d \geq 3$ and $X_{i+2} \neq \emptyset$, we conclude that $\left|X_{i+2} \cup X_{i+d}\right|>\frac{\alpha}{2}$. However $X_{i+2} \cup X_{i+d} \subseteq Y_{i+1} \cap Y_{i+2}$. So $Y_{i+1}$ and $Y_{i+2}$ are two distinct maximum stable sets with $\left|Y_{i+1} \cap Y_{i+2}\right|>\frac{\alpha}{2}$, in contrary to Lemma 7.

Thus for every $i \in \mathbb{Z}_{k}$, we have $\left|Y_{i}\right|+\left|Y_{i+1}\right|+\left|Y_{i+2}\right|<3 \alpha$. Since $\sum_{i \in \mathbb{Z}_{k}}\left|Y_{i}\right|=$ $d n$, we have $\sum_{i \in \mathbb{Z}_{k}}\left(\left|Y_{i}\right|+\left|Y_{i+1}\right|+\left|Y_{i+2}\right|\right)=3 d n \leq(3 \alpha-1) k$.

As $n=\alpha \omega+1$ and $\frac{k}{d}<\omega+1$, we have

$$
3 n=3(\alpha \omega+1)<(3 \alpha-1)(\omega+1) .
$$

It follows that $\omega<3 \alpha-4$. Hence

$$
2^{p+1} \delta \leq \omega<3 \alpha-4
$$

which is a contradiction, as $\alpha=2^{p}$ and $\delta \geq 2$.

By Lemma 8, we have $d=1$ or 2 . If $d=1$, we are done. Thus we assume $d=2$, and we shall derive a contradiction. As $\omega<\chi_{c}(G) \leq \chi(G)=\omega+1$ and $d=2$, we have $k=2 \omega+1$. 
Lemma 9 There exists $i \in \mathbb{Z}_{k}$ such that $Y_{i}, Y_{i+1}, \ldots, Y_{i+2 \delta}$ are all of size $\alpha$.

\section{PROOF.}

- Case 1: $p=2$

If there exists $i \in \mathbb{Z}_{k}$ such that $\left|X_{i}\right| \geq 3$ then $Y_{i}$ and $Y_{i-1}$ are two maximum stable sets sharing $\left|X_{i}\right|>\alpha / 2$ vertices, in contrary to Lemma 7. Thus, for every $i \in \mathbb{Z}_{k}$, we have $\left|X_{i}\right| \leq 2$. Since $\sum_{i \in \mathbb{Z}_{k}}\left|X_{i}\right|=n=4 x_{1} x_{2}+1=$ $2 k-1$, it follows that there exists an unique $j \in \mathbb{Z}_{k}$, such that $\left|X_{j}\right|=1$. Thus $Y_{j+1}, Y_{j+2}, \ldots, Y_{j+n-2}$ are $n-2$ stable sets of size $\alpha=4$. As $n-2=4 x_{1} x_{2}-1>$ $2 \delta+1$, we are done.

- Case 2: $\omega \geq 2^{p+1} \delta$

Assume to the contrary that for every $i \in \mathbb{Z}_{k}$, there exists one stable set in $Y_{i}, Y_{i+1}, \ldots, Y_{i+2 \delta}$ of size strictly less than $\alpha$, then

$$
\sum_{i=0}^{k-1}\left(\left|Y_{i}\right|+\left|Y_{i+1}\right|+\cdots+\left|Y_{i+2 \delta}\right|\right) \leq((2 \delta+1) \alpha-1) k .
$$

On the other hand, as $\sum_{i=0}^{k-1}\left|Y_{i}\right|=2 n$, we have

$$
\sum_{i=0}^{k}\left(\left|Y_{i}\right|+\left|Y_{i+1}\right|+\cdots+\left|Y_{i+2 \delta}\right|\right)=(2 \delta+1) 2 n
$$

Therefore

$$
(2 \delta+1) 2 n \leq((2 \delta+1) \alpha-1) k .
$$

Since $k=2 \omega+1$ and $n=\omega \alpha+1$, straightforward calculation shows that

$$
4 \delta+2 \leq(2 \delta+1) \alpha-2 \omega-1
$$

As $\omega \geq 2^{p+1} \delta$ and $\alpha=2^{p}$, easy calculation derives a contradiction.

In the remainder of this section, let $i$ be an index such that $Y_{i}, Y_{i+1}, \ldots, Y_{i+2 \delta}$ are all of size $\alpha$.

Lemma 10 For every $j=i+1, i+2, \ldots, i+2 \delta$, we have $\left|X_{j}\right|=\frac{\alpha}{2}$.

PROOF. By Lemma 7, $\left|X_{j}\right|=\left|Y_{j-1} \cap Y_{j}\right| \leq \frac{\alpha}{2}$. As $\alpha=\left|Y_{j}\right|=\left|X_{j}\right|+\left|X_{j+1}\right|$, it follows that $\left|X_{j}\right|=\alpha-\left|X_{j+1}\right| \geq \frac{\alpha}{2}$ if $i+1 \leq j \leq i+2 \delta-1$. Hence $\left|X_{j}\right|=\frac{\alpha}{2}$. If $j=i+2 \delta$, then since $\alpha=\left|Y_{j-1}\right|=\left|X_{j-1}\right|+\left|X_{j}\right|$, we also have $\left|X_{j}\right|=\frac{\alpha}{2}$. 
Lemma 11 There exists an index $t \in\{1,2, \cdots, p\}$ and $a \in \mathbb{Z}_{n}$, such that either for all $j=1,2, \ldots, 2 \delta$,

$$
X_{i+j}=X_{i}+j c_{t}=B_{t}+a+j c_{t}, \text { and } Y_{i+j-1}=S+a+(j-1) c_{t}
$$

or for all $j=1,2, \ldots, 2 \delta$,

$$
X_{i+j}=X_{i}-j c_{t}=B_{t}+a-j c_{t} \text {, and } Y_{i+j-1}=S+a-(j-1) c_{t} .
$$

\section{PROOF.}

Let $a$ be the element in $\mathbb{Z}_{n}$ such that $Y_{i}=S+a$.

By definition, for any $1 \leq t \leq p, S=B_{t}+\left\{0, c_{t}\right\}=B_{t} \cup\left(B_{t}+c_{t}\right)$. By Lemma 10, $\left|Y_{i} \cap Y_{i+1}\right|=\frac{\alpha}{2}$. By Lemma 7, $Y_{i+1}=Y_{i} \pm c_{t}$ for some $1 \leq t \leq p$. First we consider the case that $Y_{i+1}=Y_{i}+c_{t}$. Since $Y_{i}=S+a=\left(B_{t}+a\right) \cup\left(B_{t}+a+c_{t}\right)$, we have

$$
X_{i+1}=\left(Y_{i} \cap Y_{i+1}\right)=\left(S \cap\left(S+c_{t}\right)\right)+a=B_{t}+c_{t}+a .
$$

Moreover,

$$
X_{i+2}=\left(Y_{i+1} \backslash Y_{i}\right)=\left(\left(S+c_{t}\right) \backslash S\right)+a=B_{t}+2 c_{t}+a
$$

Assume $3 \leq j \leq 2 \delta, X_{i+j-1}=B_{t}+a+(j-1) c_{t}$ and $Y_{i+j-2}=S+a+(j-2) c_{t}$.

Assume $Y_{i+j-1}=S+j^{\prime}$ for some $j^{\prime} \in \mathbb{Z}_{n}$. Since

$$
Y_{i+j-2}=S+a+(j-2) c_{t}
$$

and

$$
Y_{i+j-1} \cap Y_{i+j-2}=X_{i+j-1}=B_{t}+a+(j-1) c_{t},
$$

it follows that

$$
S \cap\left(S+\left(j^{\prime}-a-(j-2) c_{t}\right)\right)=B_{t}+c_{t} .
$$

By Lemma 7, $j^{\prime}-a-(j-2) c_{t}=c_{t}$. Thus we conclude that $Y_{i+j-1}=S+a+(j-1) c_{t}$ and $X_{i+j}=B_{t}+a+j c_{t}$, as $X_{i+j}=Y_{i+j-1} \backslash X_{i+j-1}$.

If $Y_{i+1}=Y_{i}-c_{t}$, then the same argument shows that for $j=1,2, \cdots, 2 \delta, X_{i+j}=$ $B_{t}+a-j c_{t}$ and $Y_{i+j-1}=S+a-(j-1) c_{t}$.

Now we derive the final contradiction. Let $t$ be the index given in Lemma 11 . Without loss of generality, we assume that for $j=1,2, \cdots, 2 \delta, X_{i+j}=B_{t}+a+j c_{t}$. If $t<p$, then $2 x_{t+1} c_{t}=c_{t+1}$. Then $X_{i+2 x_{t+1}}=X_{i}+2 x_{t+1} c_{t}=B_{t}+a+c_{t+1}$. By definition, $c_{t+1} \in B_{t}$. Thus $a+c_{t+1} \in B_{t}+a=X_{i}$. On the other hand, $0 \in B_{t}$, 
and hence $a+c_{t+1} \in X_{i+2 x_{t+1}}$. This is a contradiction, as $2 x_{t+1} \leq 2 \delta<\omega<k$, which implies that $X_{i} \cap X_{i+2 x_{t+1}}=\emptyset$. If $t=p$, then

$$
2 x_{1} c_{t}=\left(2^{p} x_{1} x_{2} \cdots x_{p}\right) x_{1}=(n-1) x_{1}=-x_{1} .
$$

Then $X_{i+2 x_{1}}=X_{i}+2 x_{1} c_{t}=B_{t}+a-x_{1}$. By definition, $x_{1} \in B_{t}$. Thus $a \in$ $B_{t}+a-x_{1}=X_{i+2 x_{1}}$. On the other hand, $0 \in B_{t}$, and hence $a \in B_{t}+a=X_{i}$. This is a contradiction, as $2 x_{1}<k$ and hence $X_{i} \cap X_{i+2 x_{1}}=\emptyset$. This completes the proof of Theorem 2 .

\section{Open question}

Theorem 2 gives the circular chromatic number of some circular partitionable graphs such that their stability number is a power of two (these graphs are said to be of type 1 or 2 in [1]).

However, we believe that our result is likely to hold for most of the circular partitionable graphs: e.g., is it true that every graph $C\left[m_{1}, m_{2}, \ldots, m_{2 r}\right]$ with $r \geq 2$ has its circular chromatic number equal to its chromatic number?

\section{References}

[1] Gábor Bacsó, Endre Boros, Vladimir Gurvich, Frédéric Maffray, and Myriam Preissmann. On minimal imperfect graphs with circular symmetry. J. Graph Theory, 29(4):209-225, 1998.

[2] C. Berge. Färbung von graphen, deren sämtliche bzw. deren ungerade kreise starr sind. Wiss. Z. Martin-Luther-Univ. Halle-Wittenberg Math.-Natur. Reihe, 10:114, 1961.

[3] R. G. Bland, H. C. Huang, and L. E. Trotter, Jr. Graphical properties related to minimal imperfection. Discrete Math., 27(1):11-22, 1979.

[4] E. Boros, V. Gurvich, and S. Hougardy. Recursive generation of partitionable graphs. J. Graph Theory, 41(4):259-285, 2002.

[5] M. Chudnovsky, N. Robertson, Seymour P., and R. Thomas. The strong perfect graph theorem. preprint, 2002.

[6] V. Chvátal, R. L. Graham, A. F. Perold, and S. H. Whitesides. Combinatorial designs related to the perfect graph conjecture. In Topics on perfect graphs, volume 88 of North-Holland Math. Stud., pages 197-206. North-Holland, Amsterdam, 1984.

[7] Guogang Gao and Xuding Zhu. Star-extremal graphs and the lexicographic product. Discrete Math., 152(1-3):147-156, 1996. 
[8] Ko-Wei Lih, Daphne Der-Fen Liu, and Xuding Zhu. Star extremal circulant graphs. SIAM J. Discrete Math., 12(4):491-499 (electronic), 1999.

[9] L. Lovász. A characterization of perfect graphs. J. Combinatorial Theory Ser. B, 13:95-98, 1972.

[10] Manfred W. Padberg. Perfect zero-one matrices. Math. Programming, 6:180-196, 1974.

[11] A. Vince. Star chromatic number. J. Graph Theory, 12(4):551-559, 1988.

[12] Xuding Zhu. Star chromatic numbers and products of graphs. J. Graph Theory, 16(6):557-569, 1992.

[13] Xuding Zhu. Circular chromatic number: a survey. Discrete Math., 229(1-3):371-410, 2001. Combinatorics, graph theory, algorithms and applications.

[14] Xuding Zhu. Circular perfect graphs. preprint, 2002.

[15] Xuding Zhu. The circular chromatic number of induced subgraphs. Journal of Combin. Th. (B), to appear. 